\title{
VARIATIONAL CHARACTERISATION OF NODAL SOLUTIONS OF A STURM-LIOUVILLE PROBLEM WITH STRONG NONLINEARITY
}

\author{
LAVOSLAV ČAKLOVIĆ \\ University of Zagreb, Croatia
}

Abstract. We consider sublinear Sturm-Liouville problem

$$
\begin{aligned}
-u^{\prime \prime}+\psi(t)|u|^{p-1} u & =\lambda u, \quad p>1, \\
u(0) & =u(1)=0
\end{aligned}
$$

where $\psi$ is positive and continuous. Using the Nehari variational technique and critical point theory we prove that for each $n \in \mathbb{N}$ there is unique (up to the sign) $n$-nodal solution of the b.v.p. which is the critical point of a restricted functional associated to the problem.

\section{Introduction and Main Result}

In this paper we consider the sublinear boundary value problem

$$
\begin{aligned}
-u^{\prime \prime}+\psi(t)|u|^{p-1} u & =\lambda u, \quad p>1, \lambda \in \mathbb{R} \\
u(0) & =u(1)=0
\end{aligned}
$$

on the bounded interval $I=[0,1]$, where $\psi: I \rightarrow \mathbb{R}$ is a continuous and positive function.

As a consequence of global bifurcation theory techniques and ideas from Berestycki ([1]) it can be proved that there exists a sequence $\lambda_{n} \in \mathbb{R}, n \in \mathbb{N}$, $\lambda_{n} \rightarrow+\infty$ such that for $\lambda_{n}<\lambda \leq \lambda_{n+1}$ exists at least $(2 n+1)$ solutions of $(1.1): u_{0}(\lambda)=0, u_{0}^{ \pm}(\lambda), \ldots, u_{n}^{ \pm}(\lambda) ; u_{j}^{ \pm}(\lambda) \in S_{j}, j=1, \ldots, n$. $S_{j}$ denotes, roughly speaking, the class of functions with exactly $j-1$ nodal points.

The existence of $n$-nodal solutions of a Sturm-Liouville problem is well studied in the literature even for more general nonlinearities and boundary

2010 Mathematics Subject Classification. 34C25, 47H15, 58E05, 58F05, 70H30.

Key words and phrases. Critical point theory, Palais-Smale condition, Sturm-Liouville problem, nodal solutions. 
conditions. All of them use, roughly speaking, the idea of merging positive (negative) solutions on intervals $(0, a)$ and $(a, 1)$ by varying $a$ in order to obtain a $C^{2}$-function, the solution on the whole interval. This idea seems to be originally used by Nehari $([13])$. The characteristic energies defined by Nehari were recognised as Ljusternik-Schnirelman levels of an auxiliary functional on an appropriate manifold by Coffman ([6]).

For sublinear problems on a compact interval, Hempel ([12]) proved the existence of nodal solutions also using a variational principle which involves the variation of nodal points and his critical levels were identified with Ljusternik-Schnirelman levels by Coffman ([7]). Sublinear problems on unbounded intervals and radially symmetric problems were studied by Heinz $([8,10])$ with the help of refined versions of Ljusternik-Schnirelman theory. A nice overview of these results is given in Chen [4], Heinz [10] and Weth [15]. We are expressing out gratitude to the unknown referee for pointing out some results which were unknown to us. Let us briefly recall some of the results related to the problem.

In Hempel [12] the following regular Sturm-Liouville problem is considered ${ }^{1}$

$$
\begin{aligned}
-\left(p(t) u^{\prime}\right)^{\prime}+f(t, u) & =\lambda r(t) u, \\
u(a)=u(b) & =0,
\end{aligned}
$$

on the interval $I=[a, b]$ with continuous $r, f$ and $p \in C^{1}(I)$, where:

(A) $p, r$ are supposed to be positive and bounded away from zero on $I$.

The function $f: I \times \mathbb{R} \rightarrow \mathbb{R}$ is supposed to satisfy the following condition:

(B) For every fixed $t \in I$ the function $u \mapsto f(t, u) / u$ is strictly increasing for $u>0$, and such that

$$
\lim _{u \rightarrow 0} f(t, u) / u=0 ; \quad \lim _{u \rightarrow+\infty} f(t, u) / u \geq r(t) .
$$

(C) $f(t,-u)=-f(t, u)$ and $u f(t, u)>0$ for every $t \in I, u \in \mathbb{R}, u \neq 0$.

The equation (1.2) is the Euler equation of a functional $\Gamma(u ; a, b)$, defined on the whole space $W_{0}^{1,2}(I)$, which is bounded from bellow and attains its infimum. The minimum point can be taken to be non-negative. The same reasoning may be applied to any sub-interval $(\alpha, \beta) \subseteq I$ and any interval $\left(a_{i}, a_{i+1}\right)$ of the subdivision

$$
a=a_{0} \leq a_{1} \leq a_{2} \leq \ldots \leq a_{n} \leq a_{n+1}=b
$$

of $I$. Let us consider $\Delta^{n}$, the set of all $n$-tuples $a=\left(a_{1}, \ldots, a_{n}\right)$ which satisfies (1.3) and $\Delta_{0}^{n}$, the subset of all sequences which satisfies strict inequalities in (1.3).

Combining the solutions of homogeneous b.v.p. on each interval $\left(a_{i}, a_{i+1}\right)$ and varying the points $a_{i}$ it is possible to construct the solution of $(1.2)$

\footnotetext{
${ }^{1}$ The original notation is slightly changed. Originally, the equation was written as $\left(a(x) u^{\prime}\right)^{\prime}+c(x) u-b(x, u)=0$.
} 
with the prescribed number of nodal points. More precisely, if we denote by $0<\lambda_{1}<\cdots<\lambda_{n}<\cdots\left(\lambda_{n} \rightarrow+\infty\right)$ the eigenvalues of the related linear eigenvalue problem

$$
\begin{aligned}
-\left(p(t) u^{\prime}\right)^{\prime} & =\lambda r(t) u, \\
u(a)=u(b) & =0,
\end{aligned}
$$

then:

Theorem (Hempel). Suppose $\lambda_{p}<\lambda \leq \lambda_{p+1}$. Then, under the conditions (A), (B), (C), the equation (1.2) has p pairs of non-trivial solutions $\left\{ \pm u_{n}(t)\right\}, n=1, \ldots, p$. The solution $u_{n}$ has exactly $n-1$ zeroes in $(a, b)$. Furthermore, there exists at most one solution of (1.2) which is positive in $(a, b)$.

Heinz ([10]) considered slightly different b.v.p.

$$
\begin{aligned}
-\left(p(t) u^{\prime}\right)^{\prime}+q(t) u+f(t, u) & =\lambda r(t) u, \\
u(a)=u(b) & =0,
\end{aligned}
$$

on interval $I=[a, b]$ with continuous $q, r, f$ and $p \in C^{1}(I)$, with (A), (B') and (C) where:

(B') For every fixed $t \in I$ the function $u \mapsto f(t, u) / u$ is strictly increasing on $\mathbb{R}^{+}$.

By adding a multiple of $r(u)$ to both sides of (1.4) we can arrange that $q(t) \geq 0$ on $I$. Moreover, without loss of generality we can suppose that $p \equiv 1$ and $q=0$. This can be achieved by a transformation $t=t(s)$ with $d s=d t / p(t)$ and by including the term $q(t) u$ into the nonlinearity $f$, which does not affect (B') and (C).

The equation (1.4) is the Euler-Lagrange equation of the functional

$$
\Phi(u):=\frac{1}{2} \int_{a}^{b} u^{\prime}(t)^{2} d t+\int_{a}^{b} N_{f}(t, u(t)) d t
$$

with the constraint

$$
\|u\|^{2}:=\int_{a}^{b} r(t) u(t)^{2} d t=R,
$$

defined on the Sobol'ev space $W_{0}^{1,2}(I)$, where $N_{f}(t, u):=\int_{0}^{u} f(t, s) d s$ and $R$ is a constant. The Niemitzky operator $N_{f}(t, u)$ is convex and even in $u$ and nonegative. $\Phi$ is convex and continuous, thus lower semicontinuous, which allows us to minimize it over the set of all functions $u$, such that $\|u\|=R$ and $u\left(a_{1}\right)=\ldots=u\left(a_{n}\right)=0$. Heinz shows $([10$, Proposition 3.4$])$ that there exists a unique non-negative minimizing function $u_{0}$ and solutions $u$ of the above b.v.p. are exactly those continuous functions such that $u\left(a_{1}\right)=\ldots=u\left(a_{n}\right)=$ 0 and $|u|=u_{0}$. The consequence of this result is the following theorem: 
Theorem (Heinz). Suppose assumptions (A), (B') and (C) are satisfied for the b.v.p problem (1.4). Let $R>0$ and $n \geq 1$ be arbitrary. Then

(a)

$$
c_{n}=\sup _{\substack{a \in \Sigma_{0}^{m} \\ m \leq n}} \inf _{\substack{u \in W_{0}^{1,2}(I) \\\|u\|=R \\ u\left(a_{i}\right)=0}} \Phi(u)
$$

(b) $c_{n}<c_{n+1}$.

(c) There exists a solution $(u, \lambda)$ of the b.v.p. problem (1.4) such that $\|u\|=R$ and $\Phi(u)=c_{n}$, and such that $u$ has precisely $n-1$ zeroes in $(a, b)$.

(d) If $u$ is an eigenfunction such that $\|u\|=R$ and $\Phi(u)>c_{n}$, then $u$ has at least $n$ zeroes in $(a, b)$.

The proof of this theorem uses the invariance of variational problem under the symmetry $u \mapsto-u$ and the Ljusternik-Schnirelman theory. The critical value $c_{n}$ from the statement (a) is obtained as a maximum of $\Phi(u)$ over some set $P_{n}$ with genus $\gamma\left(P_{n}\right)=n$.

In his later work [9] Heinz studied the sublinear Sturm-Liouville equation (1.1) on unbounded domain $I=(a, \infty)$ with boundary conditions:

$$
u(0)=0, u^{\prime}(0)=\xi .
$$

The author investigates the dependence of the $n$-th root $x_{n}(\xi, \lambda)$ of the solution on unbounded domain as a function of $\xi$. If $\psi$ satisfies the condition of logarithmic convexity, i.e., $\psi^{\prime} / \psi$ is nondecreasing on $I$, then

$$
\begin{aligned}
\lim _{\xi \rightarrow 0} x_{n}(\xi, \lambda) & =n \pi / \sqrt{\lambda} \\
\lim _{\xi \rightarrow \omega_{n}} x_{n}(\xi, \lambda) & =+\infty
\end{aligned}
$$

for some $0<\omega_{n} \leq \infty$. Then ([9, Theorem 4.1.]), for the bounded interval $I=(a, b)$, it is evident that there exists $\xi$ such that $x_{n}(\xi, \lambda)=b$ and:

(a) for $\lambda \leq \lambda_{n}$ there is no solution with $n$ nodal points on $I=(a, b)$, and for $\lambda>\lambda_{n}$ there are exactly two solutions $\pm u_{n, \lambda}, u_{n, \lambda}^{\prime}(0)>0$, with $n$ nodal points,

(b) $\left(u_{n, \lambda}, \lambda\right)_{\lambda>\lambda_{n}}$ is a $C^{1}$ curve in the Banach space $C^{1}[a, b] \times \mathbb{R}$ which bifurcates from $(\lambda, u)=(0,0)$.

The logarithmic convexity of $\psi$ is sufficient for the existence of $n$-nodal solution because of the technique developed for unbounded domain.

The critical values $c_{n}$ in the result of Heinz [10] are not related to the eigenvalues $\lambda_{n}$ of the linearised problem, while $n$-nodal solutions in [9] are not related to the critical levels of some functional. On the other side, Hempel has $n$-nodal solution $u_{n}$ if $\lambda_{n}<\lambda$. Although $\Gamma\left(u_{n} ; a, b\right)$ has a variational characterisation, see [12, Theorem 5., eq (4.4)], the $n$-nodal solutions of 
Hempel are not directly related to the critical points of some functional. We use a slightly different variational technique to 'unify' the results of Hempel and Heinz (Theorem 6.3), but for a more restricted nonlinearity of the form

$$
f(t, u)=\psi(t)|u|^{p-1} u
$$

where $p>1$ and $\psi: I \rightarrow \mathbb{R}^{+}$is continuous.

Instead of the functional given in (1.5) we consider

$$
\Phi(u):=\frac{1}{2} \int_{0}^{1} u^{\prime}(t)^{2} d t-\frac{\lambda}{2} \int_{0}^{1} u(t)^{2} d t, \quad u \in W_{0}^{1,2}(I),
$$

the functional $\Psi$

$$
\Psi(u):=\frac{1}{p+1} \int_{0}^{1} \psi(t)|u(t)|^{p-1} u d t
$$

and the sets

$$
\begin{aligned}
& \Omega:=\{u \in H \mid \Psi(u) \leq 1\}, \\
& \Sigma:=\partial \Omega=\{u \in H \mid \Psi(u)=1\} .
\end{aligned}
$$

The restriction $\left.\Phi\right|_{\Sigma}$ is a $C^{1}$-function on $\Sigma$ which satisfies the (PS)-condition of Palais-Smale and the critical points of $\left.\Phi\right|_{\Sigma}$ lead to the solution of the b.v.p., cf. Lemma 3.1. Without loss of generality we suppose that $r \equiv 1$ and $I=(0,1)$. For a subdivision $a \in \Delta^{n}$ let us denote

$$
\Sigma_{a}:=\left\{u \in \Sigma^{n} \mid u\left(a_{i}\right)=0, i=1, \ldots, n\right\} .
$$

The restriction $\left.\Phi\right|_{\Sigma_{a}}$ is bounded below, satisfies the (PS)-condition and attains the minimum. Let us denote by $u_{a} \in \Sigma_{a}$ the minimum point, i.e.,

$$
\Phi\left(u_{a}\right)=\min _{u \in \Sigma_{a}} \Phi(u) .
$$

The following Lemma and Theorem will be proved later in section 6 .

LEMmA 6.2. The function $a \mapsto \Phi\left(u_{a}\right)$ is continuous on $\Delta^{n}$ and attains its maximum at the interior point of $\Delta_{0}^{n}$.

TheOrem 6.3. Let us define

$$
C_{n}:=\max _{a \in \Delta_{0}^{n}} \Phi\left(u_{a}\right)
$$

Assume $\lambda>\lambda_{n+1}$. Then,

i) $C_{n}$ is the critical value of $\left.\Phi\right|_{\Sigma}$ and $C_{n-1}<C_{n}<0 \quad \forall n \in \mathbb{N}$.

ii) The critical point $u_{a}$ changes the sign at each nodal point and it is unique up to the sign. 
Theorem 6.3 is slightly more precise than a theorem of Heinz ([10, Theorem 4.2]) because the existence of the nodal solution with the prescribed number of nodal points explicitly depends on $\lambda$, i.e., on $\lambda>\lambda_{n+1}$. The part (d) of the theorem of Heinz has its counterpart in Theorem 6.4.

Among the authors who have studied the solutions of equation (1.1) on the half line $I=(a,+\infty)$ let us mention Chen $([2,3])$ and Heinz $([11])$. In $[2]$ the author studied the equation (1.2) with more general boundary condition

$$
u(a) \cos \theta-u^{\prime}(a) \sin \theta=0, \quad u \in L^{2}(I)
$$

$0 \leq \theta \leq \pi / 2$. The nonlinearity $f(t, u)$ satisfies less restrictive assumptions than (B), may not be odd and is bounded below, i.e., (D) $f(t, u) \geq \omega(t)|u|^{\sigma}$ with some additional growth condition on $\omega^{2}$. The results of Chen are generalisations of the result of Heinz for $\theta=0$ and can be applied to a bounded domain, but the above restriction (D) on the non-linearity still remains. Thanks to a different approach to the problem, Theorem 6.3 in this article assumes only the positivity of $\psi$.

The paper is organised as follows. Some basic facts from the classical literature are stated in Section 2. The variational framework of the b.v.p. is set in Section 3. The existence and uniqueness of positive solution are proved in Section 4. The main part of the article is the Section 5 where the existence of 1-nodal solution is proved. The construction of multiple nodalpoints is given in Section 6, while some special cases are considered in Section 7. A comment about superlinear problem is given in the last section and the Appendix contains the classical deformation lemma of Rabinowitz from the critical point theory.

\section{Some Basic FACts}

Let us denote by $S_{k}, k \in \mathbb{N}$, the set of all continuous functions which have $k-1$ simple zeroes in the open interval $I=(0,1)$. This kind of zeroes we call nodal points. By nodal solution we understand a solution with zeroes that are nodal points. Let us state some facts about the linear equation which we shall need later. The proof can be found in the book of Coddington-Levinson $[5]$.

Consider the linear eigenvalue problem

$$
-\varphi^{\prime \prime}+q \varphi=\lambda \varphi
$$

where $q:[0,1] \rightarrow \mathbb{R}$ is a positive continuous function. By $\lambda_{k}(q)$ we denote the $k$-th eigenvalue, $k \in \mathbb{N}$, and by $\varphi_{k}$ the corresponding eigenfunction. The function $\varphi_{k}$ has $k-1$ nodal points, i.e., $\varphi_{k} \in S_{k}$.

If $q \equiv 0$ then we write $\lambda_{k}:=\lambda_{k}(0)$. It is not difficult to see that $\lambda_{k}=k^{2} \pi^{2}$. If we denote by $\lambda_{k}(a, b)$ the eigenvalues on the interval $(a, b)$, then $\lambda_{k}(a, b)=$

\footnotetext{
${ }^{2}$ In fact we need two growth conditions: one for negative and another for positive values of $u$.
} 
$\frac{\lambda_{k}}{(b-a)^{2}}$. The following Lemma is a consequence of the classical variational characterisation of the eigenvalues of a linear Sturm-Liouville problem.

Lemma 2.1. Assume $q_{1} \leq q_{2}$. Then, $\lambda_{k}\left(q_{1}\right) \leq \lambda_{k}\left(q_{2}\right)$ for all $k \in \mathbb{N}$ with strict inequality if $q_{1} \not \equiv q_{2}$.

\section{Variational Formulation of The Problem}

Let us denote the norm on the Sobol'ev space $H:=W_{0}^{1,2}(0,1)$ by

$$
\|u\|=\left(\int_{0}^{1}\left|u^{\prime}(t)\right|^{2} d t\right)^{1 / 2}
$$

and the scalar product by

$$
\langle u \mid v\rangle=\int_{0}^{1} u^{\prime}(t) v^{\prime}(t) d t .
$$

The restriction $\left.\Phi\right|_{\Sigma}$ is a $C^{1}$ function on $\Sigma$ and the directional derivative is

$$
\begin{aligned}
\left(\left.\Phi\right|_{\Sigma}\right)^{\prime}(u) v= & \Phi^{\prime}(u) v-\alpha \Psi^{\prime}(u) v \\
= & \int_{0}^{1} u^{\prime}(t) v^{\prime}(t) d t-\lambda \int_{0}^{1} u(t) v(t) d t \\
& -\alpha \int_{0}^{1}|\psi(t)||u(t)|^{p-1} u(t) v(t) d t
\end{aligned}
$$

for some $\alpha \in \mathbb{R}$ and $v \in H$.

Lemma 3.1. Suppose $u \in \Sigma$ is a critical point of $\left.\Phi\right|_{\Sigma}$ with negative (positive) critical value $\Phi(u)$. Then $v=\left[\frac{2 \Phi(u)}{p+1}\right]^{\frac{1}{p-1}} u$ is a weak solution of equation (1.1) for positive (negative) $\psi$.

Proof. For $v \in H$

$$
\begin{aligned}
\left(\left.\Phi\right|_{\Sigma}\right)^{\prime}(u) v & =\int_{I} u^{\prime}(t) v^{\prime}(t) d t-\lambda \int_{I} u(t) v(t) d t \\
& -\alpha \int_{I}|\psi(t)||u(t)|^{p-1} u(t) v(t) d t
\end{aligned}
$$

Then, $\left(\left.\Phi\right|_{\Sigma}\right)^{\prime}(u) u=0$ and $\alpha=\frac{2 \Phi(u)}{p+1}$ which implies that $u$ is a weak solution of equation

$$
-u^{\prime \prime}=\lambda u+\alpha(u)|\psi(t)||u|^{p-1} u .
$$

If we take $v=(\alpha)^{\frac{1}{p-1}} u$, then $v$ is a weak solution of (1.1).

Using the bootstrap type arguments it follows that any weak solution is also a classical solution. 
3.1. PS-condition. We say that a functional $\Phi: H \rightarrow \mathbb{R}$ satisfy (PS)condition if

$$
\left.\begin{array}{l}
\left|\Phi\left(u_{n}\right)\right| \leq \text { const. } \\
\Phi^{\prime}\left(u_{n}\right) \rightarrow 0
\end{array}\right\} \Longrightarrow\left\{\Phi\left(u_{n}\right)\right\} \text { has a convergent subsequence. }
$$

LEMMA 3.2. $\left.\Phi\right|_{\Sigma}$ is bounded from below and satisfies (PS)-condition.

Proof. Using Poincaré's inequality we obtain

$$
2 \Phi(u) \geq\left(\lambda_{1}-\lambda\right) \int_{I} u^{2}(t) d t .
$$

The boundedness of $\Phi$ follows from the fact that $\Sigma$ is bounded in $L^{2}$-norm.

To prove (PS)-condition, let us suppose that for a sequence $\left(u_{n}\right) \subset \Sigma$ we have $\left|\Phi\left(u_{n}\right)\right| \leq C$ and $\left(\left.\Phi\right|_{\Sigma}\right)^{\prime}\left(u_{n}\right) \rightarrow 0$. This means that

and therefore

$$
-C \leq \int_{I}\left|u_{n}^{\prime}(t)\right|^{2} d t-\lambda \int_{I} u_{n}^{2}(t) d t \leq C
$$

$$
\begin{aligned}
\int_{I}\left|u_{n}^{\prime}\right|^{2}(t) d t & \leq C+\lambda \int_{I} u_{n}^{2}(t) d t \\
& \leq C+\lambda K \int_{I}\left|u_{n}(t)\right|^{p+1} d t \\
& =C+\lambda K,
\end{aligned}
$$

where $K$ is a constant. Then

$$
\varepsilon_{n}:=\left(\left.\Phi\right|_{\Sigma}\right)^{\prime}\left(u_{n}\right) u_{n} \rightarrow 0
$$

because $\left(u_{n}\right)$ is a bounded sequence. This implies that

(3.2) $\varepsilon_{n}=\int_{I}\left|u_{n}^{\prime}(t)\right|^{2} d t-\lambda \int_{I} u_{n}(t)^{2} d t-\alpha\left(u_{n}\right) \cdot \int_{I}|\psi(t)|\left|u_{n}(t)\right|^{p+1} d t \rightarrow 0$.

From (3.1) we conclude that $u_{n} \rightarrow u$ weakly in $H$, after passing to a subsequence if necessary. From (3.2), we conclude that $\alpha\left(u_{n}\right)$ is bounded, and passing to a subsequence again, $\alpha\left(u_{n}\right) \rightarrow \alpha$ and $\left\|u_{n}\right\| \rightarrow \alpha \cdot(p+1)+\lambda$. $\lim _{+\infty} \int_{B}\left\|u_{n}\right\|^{2} d t$, which implies strong convergence $u_{n} \rightarrow u$.

\section{Existence and Uniqueness of a Positive Solution}

The following necessary condition for existence is a direct consequence of Lemma 2.1.

LEMma 4.1. If $u \neq \equiv 0$ is a solution of the b.v.p. (1.1) then $u \in S_{k}$ for some $k \in \mathbb{N}$. If $u \in S_{k}$ is a solution of (1.1) for some $k \in \mathbb{N}$ then, $\lambda_{k}<\lambda$.

Proof. The first claim is a consequence of the uniqueness of the Cauchy problem. For the proof of the second claim let us denote $q(t):=\psi(t)|u(t)|^{p-1}$. Then $0 \leq q, q \neq 0$ which implies $\lambda=\lambda(q)$ and $\lambda_{k}=\lambda_{k}(0)<\lambda_{k}(q)=\lambda$. 
Lemma 4.2. Suppose that $\lambda \leq \lambda_{1}$. Then (1.1) has only a trivial solution.

Proof. Let $u \not \equiv 0$ be a solution of (1.1). Then $u \in S_{k}$ for some $k \in \mathbb{N}$ and $\lambda_{k}<\lambda$ according to Lemma 4.1 which contradicts the supposition.

THEOREM 4.3. Suppose that $\lambda>\lambda_{1}$. Then $\left.\Phi\right|_{\Sigma}$ attains its minimum at a function $u_{0} \in \Sigma, \Phi\left(u_{0}\right)<0$, and $u_{0}$ does not change the sign.

Proof. The minimum is attained because $\left.\Phi\right|_{\Sigma}$ is bounded from below and satisfies (PS)-condition. To see that the minimum is negative, it suffices to find a function $u \in \Sigma$ such that $\Phi(u)<0$, which is true for the first eigenfunction of $-u^{\prime \prime}$ because $\lambda>\lambda_{1}$.

On the other side, if $u_{0} \in \Sigma$ is a minimum point of $\left.\Phi\right|_{\Sigma}$ then the absolute value $\left|u_{0}\right|$ is also a minimum point of $\left.\Phi\right|_{\Sigma}$. In particular, $\left|u_{0}\right|$ is the classical solution, in particular a $C^{1}$ - function, which is possible only if $u_{0}$ does not change the sign.

Uniqueness of positive solution is the consequence of the fact that the function

$$
u \mapsto \frac{f(t, u)}{u} \text { is strictly increasing for } u>0,
$$

because in our case $f(t, u)=\psi(t)|u|^{p-1} u, p>1$ and $\psi(t)>0$. The prove that we can follow the reasoning in the proof of Lemma 4.1. in the article of Hempel [12] or apply the following theorem stated in Berestycki, [1].

THEOREM 4.4 (H. Berestycki). Let us consider the BVP

$$
\left\{\begin{aligned}
-\Delta u & =f(x, u) \text { in } B \\
u & =0 \text { on } \partial B
\end{aligned}\right.
$$

where $B \subset \mathbb{R}^{n}$ is a unit ball, $f: B \times \mathbb{R} \rightarrow \mathbb{R}$ is continuous and

i) $f(x, 0)=0, x \in B$,

ii) $s \mapsto \frac{f(x, s)}{s}$ is strictly increasing function for $s>0$,

iii) $(\forall M>0)(\exists K=K(M)>0)$ such that $s \mapsto f(x, s)+K s$ is increasing on $[0, M]$.

Then the (BVP) has at most one positive solution.

\section{Solution With One Nodal Point}

Let $a \in(0,1)$ and let us denote

$$
M_{a}=\{u \in H \mid u(a)=0\} .
$$

$M_{a}$ is a subspace in $H$ of codimension 1 and its orthogonal complement is spanned by a function $w_{a}:[0,1] \rightarrow \mathbb{R}$ defined by

$$
w_{a}(t)= \begin{cases}\frac{x}{a}, & 0 \leq x \leq a \\ \frac{1-x}{1-a}, & a \leq x \leq 1\end{cases}
$$


This is a consequence of the following consideration. From

$$
\left\langle w_{a} \mid u\right\rangle=0, \forall u \in H_{a}
$$

we conclude that

$$
\int_{I} w_{a}^{\prime \prime}(t) u(t) d t=0, \forall u \in C_{0}^{\infty}(0,1) \cap H_{a},
$$

which implies that $w_{a}^{\prime}=$ const. on the intervals $(0, a)$ and $(a, 1)$. Because of the continuity we can take $w_{a}(a)=1$.

Let us denote $\Sigma_{a}:=\Sigma \cap M_{a} . \Sigma_{a}$ is a $C^{1}$-manifold, $\left.\Phi\right|_{\Sigma_{a}}$ is also bounded from below and $\left.\Phi\right|_{\Sigma_{a}}$ satisfies (PS)-condition. The proof of these facts is exactly the same as for $\left.\Phi\right|_{\Sigma}$. Thus, there exists $u_{a} \in \Sigma_{a}$ such that

$$
\Phi\left(u_{a}\right)=\min _{u \in \Sigma_{a}} \Phi(u) .
$$

Evidently, $u_{a}$ is a critical point of $\left.\Phi\right|_{\Sigma_{a}}$ and it satisfies the equation (1.1) on each interval $(0, a)$ and $(a, 1)$ with homogeneous Dirichlet's conditions.

LEMMA 5.1. $u_{a}$ is a critical point of $\left.\Phi\right|_{\Sigma}$ if and only if

$$
u_{a}^{\prime}(a+)=u_{a}^{\prime}(a-)
$$

where $u_{a}^{\prime}(a+)$ denotes the limit of $u_{a}^{\prime}(t)$ when $t \downarrow a$, and $u_{a}^{\prime}(a-)$ denotes the limit of $u_{a}^{\prime}(t)$ when $t \uparrow a$.

Proof. By definition, $u_{a} \in \Sigma_{a}$ is a critical point of $\left.\Phi\right|_{\Sigma_{a}}$ which means that

$$
\left(\left.\Phi\right|_{\Sigma_{a}}\right)^{\prime}\left(u_{a}\right) v=0, \quad \forall v \in T_{u_{a}}\left(\Sigma_{a}\right)
$$

where $T_{u_{a}}\left(\Sigma_{a}\right)$ denotes the tangent space of $\Sigma_{a}$ at the point $u_{a}$. On the other side, $\forall v \in H$

$$
\begin{aligned}
\left(\left.\Phi\right|_{\Sigma_{a}}\right)^{\prime}\left(u_{a}\right) v & =\Phi^{\prime}\left(u_{a}\right) v-\alpha \int_{I}|\psi(t)|\left|u_{a}(t)\right|^{p-1} u_{a}(t) v(t) d t \\
& -\beta \int_{I} w_{a}^{\prime}(t) v^{\prime}(t) d t,
\end{aligned}
$$

for some $\alpha, \beta \in \mathbb{R}$; and $u_{a}$ is a critical point of $\left.\Phi\right|_{\Sigma}$ if and only if $\beta=0$. In particular, if $v \in T_{u_{a}}(\Sigma)$, then, using partial integration, the fact that $w_{a}^{\prime}(a-)=\frac{1}{a}$ and $w_{a}^{\prime}(a+)=\frac{1}{a-1}$ we obtain that critical point $u_{a}$ of $\left.\Phi\right|_{\Sigma_{a}}$ satisfies

$$
\begin{aligned}
\left(-u_{a}^{\prime}(a+)+u_{a}^{\prime}(a-)\right) v(a) & =\beta\left(\psi_{a}^{\prime}(a-)-\psi_{a}^{\prime}(a+)\right) v(a) \\
& =\frac{\beta v(a)}{a(1-a)} .
\end{aligned}
$$

This identity implies that $u_{a}$ is a critical point of $\left.\Phi\right|_{\Sigma}$ if and only if (5.2) takes place. 
LEMmA 5.2. The function $a \mapsto \Phi\left(u_{a}\right)=\min _{u \in \Sigma_{a}} \Phi(u)$ is a continuous function from $[0,1]$ to $\mathbb{R}$.

Proof. We shall prove that the function is upper semi-continuous and lower semi-continuous at each interior point and at the boundary.

(i) Upper semi-continuity. Fix $a \in(0,1)$ and $u_{a} \in \Sigma_{a}, \Phi\left(u_{a}\right)=\min _{u \in \Sigma_{a}} \Phi(u)$. $1^{\text {st }}$ step: $\forall \varepsilon>0, \exists \delta>0,\left(|a-b|<\delta \Rightarrow \exists v \in M_{b}\right.$ s.t. $\left.\left\|u_{a}-v\right\|<\varepsilon\right)$.

To prove this, let us denote by $P_{b}: H \rightarrow M_{b}$ the orthogonal projector on $M_{b}$. Then, it is sufficient to prove that $\lim _{b \rightarrow a} P_{b} u_{a}=u_{a}$. Evidently,

$$
\left\|P_{b} u_{a}-u_{a}\right\|=\frac{\left\langle u_{a} \mid \psi_{b}\right\rangle}{\left\|\psi_{b}\right\|} \rightarrow \frac{\left\langle u_{a} \mid w_{a}\right\rangle}{\left\|w_{a}\right\|}=0
$$

because $b \mapsto \psi_{b}$ is continuous at $a$ and $\left\langle u_{a} \mid w_{a}\right\rangle=0$.

$2^{\text {nd }}$ step: $\forall \varepsilon>0, \exists \delta>0\left(|a-b|<\delta \Rightarrow \exists v_{b} \in \Sigma_{b}\right.$ s.t. $\left.\left\|u_{a}-v_{b}\right\|<\varepsilon\right)$.

Let us take $v \in M_{b}, v=P_{b} u_{a}$ and let $v_{b}$ be the radial projection of $v$ on $\Sigma$. The radial projection exists and is unique because of the convexity of $\Omega$. We shall prove that $\left\|u_{a}-v_{b}\right\| \rightarrow 0$ when $\delta \rightarrow 0$. We claim that for $\delta>0$ sufficiently small

$$
\Psi^{\prime}\left(u_{a}\right) v \neq 0
$$

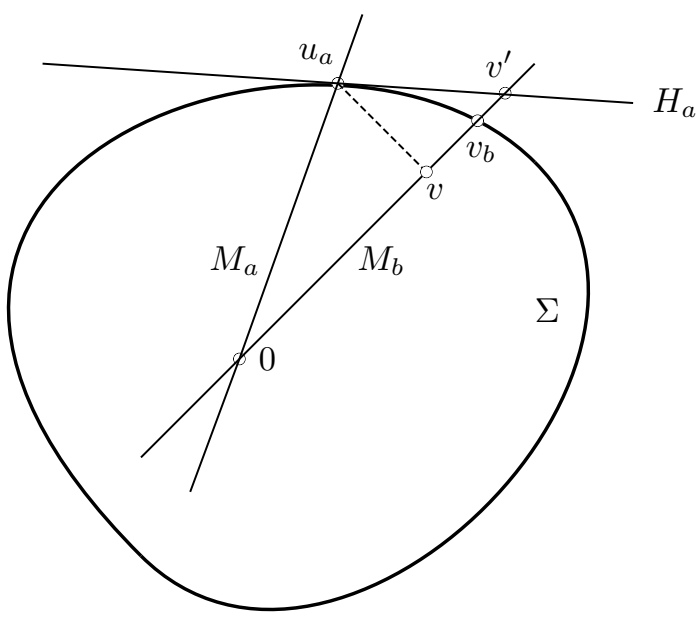

Figure 1

This is true because

$$
\Psi^{\prime}\left(u_{a}\right) v=\int_{I}|\psi(t)|\left|u_{a}(t)\right|^{p-1} u_{a}(t) v(t) d t
$$

and when $v \rightarrow u_{a}$ then $\Psi^{\prime}\left(u_{a}\right) v \rightarrow 1$. 
This means that the tangent hyperplane $H_{a}$ on $\Sigma_{a}$ at a point $u_{a}$ intersects the line generated by $v$. Let us denote this intersection by $v^{\prime}$. Then, evidently $v^{\prime}$ and $v_{b}$ approach $u_{a}$ when $b \rightarrow a$.

$3^{\text {rd }}$ step: Conclusion. Evidently, because $v_{b} \in \Sigma_{b}$,

$$
\begin{aligned}
\Phi\left(u_{b}\right) & \leq \Phi\left(v_{b}\right) \\
& =\Phi\left(v_{b}\right)-\Phi\left(u_{a}\right)+\Phi\left(u_{a}\right) \\
& \leq \Phi\left(u_{a}\right)+\varepsilon
\end{aligned}
$$

whenever $|b-a|<\delta$ which proves upper semi-continuity.

(ii) Lower semi-continuity. We are going to prove that

$$
\Phi\left(u_{a}\right) \leq \liminf _{n \rightarrow \infty} \Phi\left(u_{a_{n}}\right)
$$

for any sequence $\left(a_{n}\right)$ such that $a_{n} \rightarrow a$. Let us take a subsequence, denote it again by $\left(a_{n}\right)$, such that $\liminf _{n \rightarrow \infty} \Phi\left(u_{a_{n}}\right)=: c$.

Because of the upper semi-continuity, $\Phi\left(u_{a_{n}}\right)$ is bounded. In the same way as in the proof of (PS)-condition, we can prove that $\left(u_{a_{n}}\right)$ is bounded in $H$ and has a weakly convergent subsequence. Let us denote it again by $\left(u_{a_{n}}\right)$, i.e., $u_{a_{n}} \rightarrow u$. Moreover, $u_{a_{n}} \rightarrow u$ strongly since $\Phi\left(u_{a_{n}}\right) \rightarrow c$ and $\int u_{a_{n}}^{2} \rightarrow \int u^{2}$ implies the norm convergence of $\left(u_{a_{n}}\right)$. Now, weak and norm convergence of $\left(u_{a_{n}}\right)$ implies strong convergence of $\left(u_{a_{n}}\right)$. Evidently $u \in \Sigma$. We claim that $u \in \Sigma_{a}$. To prove this it is enough to see that the scalar product $\left\langle u, w_{a}\right\rangle=0$. This is obvious because $w_{a_{n}} \rightarrow w_{a}$ and

$$
\left\langle u \mid w_{a}\right\rangle=\lim _{n \rightarrow \infty}\left\langle u_{a_{n}} \mid w_{a_{n}}\right\rangle=0 .
$$

Finally, by the definition of $u_{a}$, we have

$$
\Phi\left(u_{a}\right) \leq \Phi(u)=c .
$$

(iii) Continuity at the boundary. We are going to prove that $\lim _{a \rightarrow 1} \Phi\left(u_{a}\right)=$ $\Phi\left(u_{0}\right)=\min _{u \in \Sigma} \Phi(u)$.

Let us define a function $v \in M_{a}$ in the following way: let $b, c \in(0,1)$ be such that $b<a<c$ and $\frac{u_{0}(c)}{c-a}=\frac{-u_{0}(b)}{b-a}>\left|u_{0}^{\prime}(1)\right|$. When $a \rightarrow 1$ then $c \rightarrow 1, b \rightarrow 1$ and $u_{0}(c) \rightarrow 0, u_{0}(b) \rightarrow 0$ because of the continuity of $u_{0}$. The numbers $b, c$ can be correctly defined when $|a-1|<\delta$ for $\delta$ sufficiently small. Let

$$
v(t)= \begin{cases}u_{0}(t), & 0 \leq x \leq b \\ -u_{0}(c) \frac{(x-a)}{c-a}, & b \leq x \leq c \\ -u_{0}(t), & c \leq x \leq 1\end{cases}
$$

If $\hat{v}$ denotes the radial projection of $v$ on $\Sigma$ then $\hat{v} \in \Sigma_{a}, \Phi(\hat{v}) \geq \Phi\left(u_{a}\right) \geq$ $\Phi\left(u_{0}\right), v \rightarrow u_{0}, \hat{v} \rightarrow u_{0}$ and $\Phi(\hat{v}) \rightarrow \Phi\left(u_{0}\right)$ whenever $a \rightarrow 1$.

A similar construction can be used to prove the continuity at the left point of the boundary. 
Lemma 5.3. The function $a \mapsto \Phi\left(u_{a}\right)$ attains its maximum at an interior point of interval $(0,1)$ and

$$
\max _{0<a<1} \Phi\left(u_{a}\right)>\min _{u \in \Sigma} \Phi(u) .
$$

Proof. The proof is a direct consequence of Lemma 5.2. If $\Phi\left(u_{a}\right)=$ $\min _{u \in \Sigma} \Phi(u)$ then $u_{a}$ cannot have a zero point which implies strict inequality.

Lemma 5.3 gives idea for construction of a solution with one nodal point. Varying $a$ we can obtain that $u_{a}$ is not only the solution of differential equation (1.1) on intervals $(0, a)$ and $(a, 1)$, with Dirichlet's boundary condition, but moreover, it satisfies the condition (5.2), i.e., $u_{a}^{\prime}(a+)=u_{a}^{\prime}(a-)$. Then it becomes a solution of the BVP (1.1). But $\lambda$ should be big enough to ensure the existence of solutions on both intervals; see the equation (5.5) below.

Theorem 5.4 (Solution with one nodal point). Assume

$$
\lambda>\lambda_{2}=\max _{0<a<1} \min \left\{\frac{\lambda_{1}}{a^{2}}, \frac{\lambda_{1}}{(1-a)^{2}}\right\} .
$$

i) Then, the number

$$
C_{1}=\max _{0<a<1} \min _{u \in \Sigma_{a}} \Phi(u)
$$

is a critical value of $\left.\Phi\right|_{\Sigma}$ and there exists a unique, up to the sign, function $u_{a}$ which is a critical point of $\left.\Phi\right|_{\Sigma}$ with $C_{1}$ as a critical value.

ii) $0>C_{1}>C_{0}:=\min _{u \in \Sigma} \Phi(u)$

Proof. ii) Let us show first that $C_{1}<0$. It suffices to prove that $\Phi\left(u_{a}\right)<0$ for all $a \in(0,1)$. Take $a \in(0,1)$. Then at least one of the inequalities $\lambda>\lambda_{1} / a^{2}, \lambda>\lambda_{1} /(1-a)^{2}$ is fulfilled. Let us suppose that $\lambda>\lambda_{1} / a^{2}$ for instance. Then there exists $u_{1} \in \Sigma$ positive on $(0, a)$ and identically equal to 0 on $[a, 1]$ and such that $\Phi\left(u_{1}\right)<0$. Evidently, $u_{1} \in \Sigma_{a}$ and

$$
\Phi\left(u_{a}\right) \leq \Phi\left(u_{1}\right)<0 .
$$

i) Uniqueness is a direct consequence of Theorem 4.4 (the uniqueness of positive solution) applied on the intervals $(0, a)$ and $(a, 1)$.

The construction of $C_{1}$ has sense because of Lemma 5.3 and there exists an interior point $a \in(0,1)$ such that

$$
C_{1}=\Phi\left(u_{a}\right)
$$

where $u_{a}$ is a minimum point of the restriction $\left.\Phi\right|_{\Sigma_{a}}$. To prove the theorem it suffices to prove that $C_{1}$ is a critical value of $\left.\Phi\right|_{\Sigma}$, and that $\Sigma_{a}$ contains a critical point of $\left.\Phi\right|_{\Sigma}$ on the $C_{1}$-level of $\left.\Phi\right|_{\Sigma}$. Let us prove that $C_{1}=\Phi\left(u_{a}\right)$ is a critical value of $\left.\Phi\right|_{\Sigma}$. Then we have two possibilities: 
First one: There exists a neighborhood of $a$ where $a \mapsto \Phi\left(u_{a}\right)$ is a constant.

Second one: $a$ is a strict local maximum of $a \mapsto \Phi\left(u_{a}\right)$.

In the first case let us assume that there exists $\delta>0$ such that $\Phi\left(u_{b}\right)=$ $C_{1}, \forall b \in(a-\delta, a+\delta)$. We claim that

$$
\left(\left.\Phi\right|_{\Sigma}\right)^{\prime}\left(u_{a}\right) v=0, \forall v \in T_{u_{a}}(\Sigma) .
$$

Using the fact that $\left(\left.\Phi\right|_{\Sigma_{a}}\right)^{\prime}\left(u_{a}\right)=0$ it is sufficient to prove only that $\left(\left.\Phi\right|_{\Sigma}\right)^{\prime}\left(u_{a}\right) w_{a}=0$. For this purpose, let us define $\gamma(t)$ by

$$
\gamma(t)=\frac{1}{p+1} \int_{I} \psi(t)\left|u_{a}(t)+t w_{a}(t)\right|^{p+1} d t, t \in \mathbb{R} .
$$

Then

$$
\left.\frac{d \gamma(t)}{d t}\right|_{t=0}=\int_{I} \psi(t)\left|u_{a}(t)\right|^{p-1} u_{a}(t) w_{a}(t) d t .
$$

On the other side, $u_{a}+t w_{a} \in \Sigma_{b}$ for some $b \in(0,1)$ and for $t$ sufficiently small we obtain

which implies

$$
0 \leq \Phi\left(\frac{u_{a}+t w_{a}}{\gamma(t)}\right)-\Phi\left(u_{a}\right)
$$

$$
\begin{aligned}
0 & \leq \lim _{t \searrow 0} \frac{1}{t}\left\{\Phi\left(\frac{u_{a}+t w_{a}}{\gamma(t)}\right)-\Phi\left(u_{a}\right)\right\} \\
& =\lim _{t \searrow 0} \frac{1}{\gamma(t)^{2}} \cdot \frac{\Phi\left(u_{a}+t w_{a}\right)-\Phi\left(u_{a}\right)}{t}+\lim _{t \searrow 0} \frac{\Phi\left(u_{a}\right)}{\gamma(t)^{2}} \cdot \frac{1-\gamma(t)^{2}}{t} \\
& =\Phi^{\prime}\left(u_{a}\right) w_{a}-2 \Phi\left(u_{a}\right) \int_{I} f(t)\left|u_{a}(t)\right|^{p-1} u_{a}(t) w_{a}(t) d t \\
& =\left(\left.\Phi\right|_{\Sigma}\right)^{\prime}\left(u_{a}\right) w_{a} .
\end{aligned}
$$

Repeating the same steps with $u_{a}-t w_{a}$ instead of $u_{a}+t w_{a}$ we obtain that $\left(\left.\Phi\right|_{\Sigma}\right)^{\prime}\left(u_{a}\right) w_{a} \leq 0$, and consequently $\left(\left.\Phi\right|_{\Sigma}\right)^{\prime}\left(u_{a}\right) w_{a}=0$.

In the second case let us take a sequence $a_{n} \rightarrow a$. We can conclude, using the same arguments as in the proof of (PS)-condition, that $\left(u_{a_{n}}\right)$ has a strongly convergent subsequence $u_{a_{n}} \rightarrow u$. Moreover, $u \in M_{a}$ because

$$
\left\langle w_{a} \mid u\right\rangle=\lim _{n \rightarrow \infty}\left\langle w_{a_{n}} \mid u_{a_{n}}\right\rangle=0,
$$

and $u$ is a critical point of $\left.\Phi\right|_{\Sigma_{a}}$ because

$$
\Phi(u)=C_{1}=\min _{v \in \Sigma_{a}} \Phi(v) .
$$

To finish the proof of the theorem we have to show that $u$ is a critical point of $\left.\Phi\right|_{\Sigma}$ or equivalently that $K_{C_{1}} \cap \Sigma_{a} \neq \emptyset$, where $K_{C_{1}}=\{v \in \Sigma \mid \Phi(v)=$ 
$\left.C_{1},\left(\left.\Phi\right|_{\Sigma}\right)^{\prime}(v)=0\right\}$ is the set of all critical points on the $C_{1}$ level. If this is not the case we shall use the Deformation Lemma to obtain a contradiction.

A preparation for Deformation Lemma is needed. Let us choose two sequences $\left(a_{n}\right),\left(b_{n}\right)$ such that $a_{n}>a, b_{n}<a, a_{n} \rightarrow a, b_{n} \rightarrow a, u_{a_{n}}(a)>$ $0, u_{b_{n}}(a)<0$ and such that $u_{a_{n}} \rightarrow u, u_{b_{n}} \rightarrow u$. This is possible because of the uniqueness of the solution (up to the sign).

Using these sequences for each $\varepsilon>0$ we can find $u^{+} \in \Sigma \cap M_{a}^{+}, u^{-} \in$ $\Sigma \cap M_{a}^{-}$, where $M_{a}^{ \pm}=\left\{u \in H \mid\left\langle w_{a} \mid u\right\rangle \gtrless 0\right\}$ are the half-spaces defined by $M_{a}$, and

$$
\Phi\left(u^{+}\right)<C_{1}-\varepsilon \text { and } \Phi\left(u^{-}\right)<C_{1}-\varepsilon .
$$

Let us define a path $\gamma:[0,1] \rightarrow \Sigma$

$$
\gamma(t):=u_{t u^{+}+(1-t) u^{-}}
$$

Evidently,

$$
\Phi(\gamma(t)) \leq C_{1}
$$

Let us now suppose that $K_{C_{1}} \cap \Sigma_{a}=\emptyset$. Because of (PS)-condition, $K_{C_{1}}$ is compact and is at a positive distance from $\Sigma_{a}$. For $C \in \mathbb{R}$, let us denote

$$
\Phi^{C}:=\{v \in \Sigma \mid \Phi(v) \leq C\}
$$

and let $\mathcal{O}$ be an open neighborhood of $K_{C_{1}}$ such that $\Sigma_{a} \cap \mathcal{O}=\emptyset$.

Then, by the Deformation Lemma (Appendix A), there exists a homeomorphism $\eta: \Sigma \rightarrow \Sigma$ such that

$$
\begin{aligned}
\eta\left(u^{+}\right) & =u^{+} \\
\eta\left(u^{-}\right) & =u^{-} \\
\eta\left(\Phi^{C_{1}+\varepsilon} \backslash \mathcal{O}\right) & \subseteq \Phi^{C_{1}-\varepsilon} .
\end{aligned}
$$

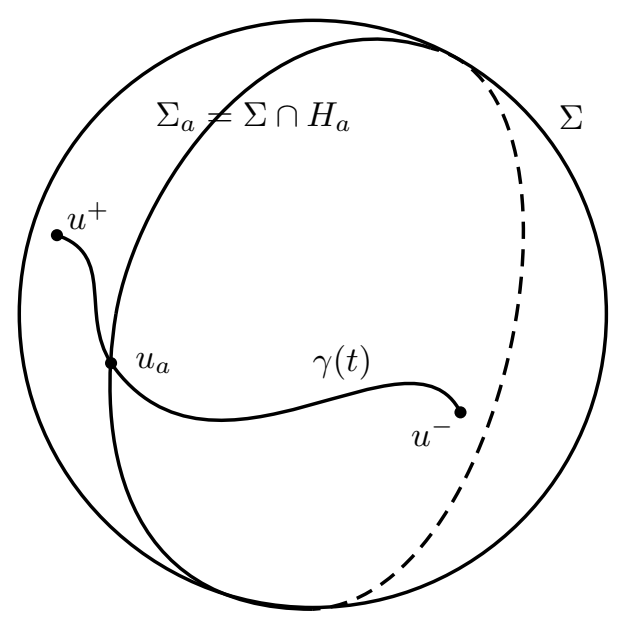

FiguRe 2 
Moreover, the path $\gamma$ can be chosen in such a way that it does not intersect $\mathcal{O}$. The set $\Sigma \backslash M_{a}$ has two components, $u^{+}$is an element of one component, and $u^{-}$is an element of another component and each path which joins $u^{+}$ and $u^{-}$has to intersect $\Sigma_{a}$ at some point. If we denote by $\Gamma$ the set of all paths $\sigma$ with the property $\sigma(0)=u^{-}, \sigma(1)=u^{+}$then

$$
\inf _{\sigma \in \Gamma} \max _{0 \leq t \leq 1} \Phi(\sigma(t)) \geq C_{1} .
$$

On the other hand $\eta(\gamma) \in \Gamma$ and

$$
\max _{0 \leq t \leq 1} \Phi(\eta(\gamma(t)))<C_{1}-\varepsilon
$$

which contradicts the previous inequality. Thus there exists $\hat{u} \in K_{C_{1}} \cap \Sigma_{a}$ such that $\Phi(\hat{u})=C_{1}$. By uniqueness of $u_{a}$ we have $\hat{u}= \pm u_{a}$, which proves the theorem.

\section{Solution With Multiple Nodal Points}

A construction of solutions with more than one nodal point follows the same procedure as the construction of the solution with one nodal point. For $n \in \mathbb{N}$ let us denote by $a$ the $n$-tuple $a=\left(a_{1}, \ldots, a_{n}\right)$ and

$$
\Delta_{0}^{n}=\left\{a \in(0,1)^{n} \mid 0=: a_{0}<a_{1}<\ldots<a_{n}<a_{n}<a_{n+1}:=1\right\} .
$$

Then, $\Delta_{0}^{n}$ is an open set in $\mathbb{R}^{n}$, and its closure we denote by $\Delta^{n}$. For $a \in \Delta_{0}^{n}$ let us denote

$$
\begin{aligned}
M_{a} & :=\left\{u \in H \mid u\left(a_{i}\right)=0, i=1, \ldots, n\right\}, \\
M_{i} & :=\left\{u \in H \mid u\left(a_{j}\right)=0, j=1, \ldots, n, j \neq i\right\}, \quad i=1, \ldots, n, \\
M_{a_{j}} & :=\left\{u \in H \mid u\left(a_{j}\right)=0\right\}, \quad j=1, \ldots, n, \\
\Sigma_{a} & :=\Sigma \cap M_{a}, \Sigma_{i}=\Sigma \cap M_{i}, \Sigma_{a_{j}}=\Sigma \cap M_{a_{j}} .
\end{aligned}
$$

$M_{a}, M_{a_{j}}, M_{i}$ are closed subspaces of $H$. The orthogonal complement $M_{a}^{\perp}$ of $M_{a}$ is spanned by

$$
M_{a}^{\perp}=\operatorname{span}\left\{w_{a_{1}}, w_{a_{2}}, \ldots, w_{a_{n}}\right\},
$$

where $w_{a_{i}}$ are defined by (5.1). Let us define also

$$
w_{i}(t)= \begin{cases}\frac{t-a_{i-1}}{a_{i}-a_{i-1}}, & a_{i-1} \leq t \leq a_{i}, \\ \frac{t-a_{i+1}}{a_{i}-a_{i+1}}, & a_{i} \leq t \leq a_{i+1}, \\ 0, & t \notin\left[a_{i-1}, a_{i+1}\right] .\end{cases}
$$

LEMMA 6.1. $M_{a}^{\perp}=\operatorname{span}\left\{w_{1}, \ldots, w_{n}\right\}$. 
Proof. Let $u \in H$. Then, an easy calculation gives for $i=1, \ldots, n$

$$
\begin{aligned}
\left\langle u \mid w_{a_{i}}\right\rangle & =\frac{u\left(a_{i}\right)}{\left(1-a_{i}\right) a_{i}}, \\
\left\langle u \mid w_{i}\right\rangle & =\frac{u\left(a_{i+1}\right)-u\left(a_{i}\right)}{a_{i}-a_{i+1}}+\frac{u\left(a_{i}\right)-u\left(a_{i-1}\right)}{a_{i}-a_{i-1}} .
\end{aligned}
$$

Obviously, $\left\langle u \mid w_{a_{i}}\right\rangle=0$ for all $i=1, \ldots, n$ which implies $\left\langle u \mid w_{i}\right\rangle=0$ for all $i=1, \ldots, n$. Let us suppose that $\left\langle u \mid w_{i}\right\rangle=0$ for all $i=1, \ldots, n$. Then

$$
\frac{u\left(a_{i}\right)-u\left(a_{i+1}\right)}{a_{i}-a_{i+1}}=\text { const. }=0, i=0, \ldots, n,
$$

and $u\left(a_{1}\right)=u\left(a_{2}\right)=\cdots=u\left(a_{n}\right)=u\left(a_{n+1}\right)=0$ which implies $\left\langle u, w_{a}\right\rangle=0$. This proves the lemma.

Let us consider now $\Sigma_{a}=M_{a} \cap \Sigma, a \in \Delta_{0}^{n}$. Then, the restriction $\left.\Phi\right|_{\Sigma_{a}}$ satisfies (PS)-condition, it is bounded below and attains its minimum. Let $u_{a} \in \Sigma_{a}$ be such that

$$
\Phi\left(u_{a}\right)=\min _{u \in \Sigma_{a}} \Phi(u) .
$$

LEMma 6.2. The function $a \mapsto \Phi\left(u_{a}\right)$ is continuous on $\Delta^{n}$ and attains its maximum at an interior point of $\Delta_{0}^{n}$.

Proof. The proof is essentially the same as the proof of Lemma 5.2 for one nodal point. There is a bit more technicality in the part of the proof that concerns continuity at the boundary.

The fact that $\Phi\left(u_{a}\right)$ attains its maximum at an interior point of $\Delta^{n}$ we prove by induction on $\operatorname{dim} \Delta^{n}$.

- Lemma 5.3 covers the case $n=1$.

- Suppose that the claim is true for dimension $n-1$ and let $a \in \Delta^{n}$ be a point of maximum value for the function $a \mapsto \Phi\left(u_{a}\right)$. Then, if there is equality in the sequence $0 \leq a_{1} \leq \ldots \leq a_{n} \leq 1$ it can occur at most once. Let it be at $i$-th place, i.e. $a_{i-1}=a_{i}$. Let us take $a_{i}^{\prime} \in\left(a_{i-1}, a_{i+1}\right)$ and define $a^{\prime}=\left(a_{1}^{\prime}, \ldots, a_{n}^{\prime}\right)$, where $a_{j}^{\prime}=a_{j}, j \neq i$.

Then, it is easy to see that $\Phi\left(u_{a}\right)<\Phi\left(u_{a^{\prime}}\right)(n=1)$, which proves that $\max _{a \in \Delta^{n}} \Phi\left(u_{a}\right)$ is attained at an interior point of $\Delta^{n}$.

TheOREM 6.3. Let us define

$$
C_{n}:=\max _{a \in \Delta_{0}^{n}} \Phi\left(u_{a}\right) .
$$

Assume $\lambda>\lambda_{n+1}$. Then,

i) $C_{n}$ is the critical value of $\left.\Phi\right|_{\Sigma}$ and $C_{n-1}<C_{n}<0 \quad \forall n \in \mathbb{N}$.

ii) The critical point $u_{a}$ changes the sign at each nodal point and it is unique up to the sign. 
Proof. i) We shall prove the claim by induction on the number of nodal points. Theorem 5.4 covers the case $n=1$.

Let $a=\left(a_{1}, \ldots, a_{n}\right) \in \Delta_{0}^{n}$ be such that $C_{n}=\Phi\left(u_{a}\right)$. By construction, $u_{a}$ is a critical point of $\left.\Phi\right|_{\Sigma_{a}}$. Furthermore, $M_{a}$ is a subspace of $M_{i}$ of codimension 1 , for each $i=1, \ldots, n$, and its orthogonal complement is spanned by $w_{i}$.

Applying now the same reasoning as in Theorem 5.4 we conclude that $u_{a}$ is a critical point of $\left.\Phi\right|_{\Sigma \cap M_{i}}, i=1, \ldots, n$. In particular

$$
\left(\left.\Phi\right|_{\Sigma_{a}}\right)^{\prime}\left(u_{a}\right) w_{i}=0, \quad i=1, \ldots, n,
$$

which is equivalent to

$$
\left(\left.\Phi\right|_{\Sigma}\right)^{\prime}\left(u_{a}\right) w_{i}=0, \quad i=1, \ldots, n .
$$

This implies that

$$
\left(\left.\Phi\right|_{\Sigma}\right)^{\prime}\left(u_{a}\right) v=0, \quad \forall v \in \operatorname{span}\left\{\psi_{1}, \ldots \psi_{n}\right\} .
$$

From Lemma 6.1 we conclude now that

$$
\left(\left.\Phi\right|_{\Sigma}\right)^{\prime}\left(u_{a}\right) v=0, \quad \forall v \in M_{a}^{\perp},
$$

which proves that $\left(\left.\Phi\right|_{\Sigma}\right)^{\prime}\left(u_{a}\right)=0$.

The inequality $C_{n-1}<C_{n}$ follows from Lemma 6.2 . Let us prove now that $C_{n}<0$. If $\lambda>\lambda_{n+1}$ then for each $a \in \Delta_{0}^{n}$

$$
\lambda>\lambda_{n+1}=\max _{a \in \Delta_{0}^{n}} \min \left\{\frac{\lambda_{1}}{a_{1}^{2}}, \frac{\lambda_{1}}{\left(a_{2}-a_{1}\right)^{2}}, \ldots, \frac{\lambda_{1}}{\left(1-a_{n}\right)^{2}}\right\}
$$

and at least one of the following inequalities is valid:

$$
\lambda>\frac{\lambda_{1}}{\left(a_{i+1}-a_{i}\right)^{2}}, i=0, \ldots, n,
$$

which implies that at least one of the integrals

$$
\int_{a_{i}}^{a_{i+1}}\left|u^{\prime}(t)\right|^{2} d t-\lambda \int_{a_{i}}^{a_{i+1}} u(t)^{2} d t, i=0, \ldots, n
$$

is negative for function $u$ defined in the following way:

$$
u(t)= \begin{cases}\varphi_{1}^{i}(t), \quad \text { for } a_{i} \leq t \leq a_{i+1} \text { if } \lambda>\frac{\lambda_{1}}{\left(a_{i+1}-a_{i}\right)^{2}} \\ \\ \text { where } \varphi_{1}^{i} \text { is the first eigenfunction } \\ \text { of }-u^{\prime \prime} \text { on }\left(a_{i}, a_{i+1}\right) \\ \text { otherwise. }\end{cases}
$$

Then $u \in \Sigma_{a}$, and $\Phi(u)<0$, implying $\Phi\left(u_{a}\right)<0$.

ii) Using the same idea as in Lemma 5.1 it is easy to see that $u_{a}$ is the critical point of $\left.\Phi\right|_{\Sigma}$ if and only if

$$
u_{a}^{\prime}\left(a_{i}+\right)=u_{a}^{\prime}\left(a_{i}-\right), \forall i=1, \ldots, n
$$


which implies that $u_{a}$ changes the sign at each nodal point. Uniqueness of $u_{a}$ follows from the uniqueness of positive (negative) solution on each interval $\left(a_{i}, a_{i+1}\right), i=0, \ldots, n$.

THEOREM 6.4. If $u \in \Sigma$ is an eigenfunction of the b.v.p. (1.1) such that $\Phi(u) \geq C_{n}$, then $u$ has at least $n$ nodal points in $(a, b)$.

Proof. On the contrary, if $u$ has $0 \leq k \leq n-1$ nodal points, then, because of Theorem 6.3

$$
\Phi(u) \leq \max _{a \in \Delta_{0}^{k}} \Phi\left(u_{a}\right)=C_{k} \leq C_{n-1}<C_{n},
$$

which contradicts the assumptions of the theorem.

\section{Some Special Cases}

Let $a \in(0,1)$ and $w, v \in H$. Let us define the functionals

$$
\begin{aligned}
\Phi_{a}(v) & =\frac{1}{2 a} \int_{0}^{1} v^{\prime}(t)^{2} d t-\frac{\lambda a^{2}}{2} \int_{0}^{1} v(t)^{2} d t \\
\hat{\Phi}_{1-a}(w) & =\frac{1}{2(1-a)} \int_{0}^{1} w^{\prime}(t)^{2} d t-\frac{\lambda(1-a)^{2}}{2} \int_{0}^{1} w(t)^{2} d t .
\end{aligned}
$$

Then, for every $u \in \Sigma_{a}$ we can write

$$
\Phi(u)=\Phi_{a}(v)+\hat{\Phi}_{1-a}(w)
$$

where $u, v, w$ are related by

$$
\begin{aligned}
v(t) & =u(a t), 0 \leq t \leq 1 \\
w(t) & =u(a+(1-a) t), 0 \leq t \leq 1 .
\end{aligned}
$$

We also have

$$
\begin{aligned}
\int_{0}^{1}|\psi(t)||u(t)|^{p+1} d t & =\left.a \int_{0}^{1}|\psi(a t) w| v(t)\right|^{p+1} d t+ \\
& +(1-a) \int_{0}^{1}|\psi(a+(1-a) t)||w(t)|^{p+1} d t .
\end{aligned}
$$

Instead of the minimisation problem

$$
\inf _{u \in \Sigma_{a}} \Phi(u)=: C_{a}
$$

we consider two minimisation problems:

$\left(\hat{\mathcal{P}}_{a-1}\right)$

$$
\begin{aligned}
\inf _{\int_{0}^{1}|\psi(a t) \| v(t)|^{p+1}=1} \Phi_{a}(v) & =: m_{a} \\
\inf _{\int_{0}^{1}|\psi(a+(1-a) t) \| w(t)|^{p+1}=1} \hat{\Phi}_{1-a}(w) & =: \hat{m}_{1-a} .
\end{aligned}
$$


Then obviously

$$
C_{a} \leq m_{a}+\hat{m}_{1-a} .
$$

LEMMA 7.1. Let us suppose that

$$
\psi\left(\frac{1+t}{2}\right)=\psi\left(\frac{t}{2}\right), t \in[0,1] .
$$

Then,

$$
2 m_{1 / 2}=C_{1 / 2} .
$$

Proof. By applying (7.3) one obtains $m_{1 / 2}=\hat{m}_{1 / 2}$ and (7.2) yields $C_{1 / 2} \leq 2 m_{1 / 2}$. On the other side $C_{1 / 2}=\Phi(u)$ for some $u \in \Sigma_{1 / 2}$. Let us define $v, w$ by (7.1). Then

$$
\begin{aligned}
C_{1 / 2} & =\Phi_{1 / 2}(v)+\hat{\Phi}_{1-1 / 2}(w) \\
& \geq 2 m_{1 / 2}
\end{aligned}
$$

which proves the lemma.

We can construct a solution $u$ of problem $(\mathcal{P})$ in the following way. Let us take a positive solution $v_{0}$ of $\left(\mathcal{P}_{1 / 2}\right)$ and define $u(t)$ by

$$
u(t)= \begin{cases}v_{0}\left(\frac{t}{a}\right), & 0 \leq t \leq a, \\ -v_{0}\left(\frac{t-a}{1-a}\right), & a \leq t \leq 1 .\end{cases}
$$

Then $u$ minimises $\left.\Phi\right|_{\Sigma_{a}}$ and it has continuous derivative at $a=1 / 2$. This implies that $u_{0}$ is a solution of the equation with one nodal point at $a=1 / 2$.

In the same way we can construct a solution with $n$ nodal points if $\psi$ satisfies

$$
\psi(t)=\psi\left(a_{i}+t\right), \quad t \in\left[0, a_{1}\right],
$$

where $a_{i}=\frac{i}{n+1}, i=1, \ldots, n$. The nodal points are precisely $a_{i}, i=1, \ldots, n$.

Corollary 7.2. Assume $\psi(t) \equiv 1$. Then for each $n \in \mathbb{N}$ there exists a unique solution of equation (1.1) with nodal points $a_{i}=\frac{i}{n+1}, i=1, \ldots, n$.

The uniqueness of nodal points is a consequence of symmetry properties of the solution with respect to the local maximum (minimum) and translationary invariance of the equation.

\section{Superlinear case}

In the superlinear case, i.e., when $\psi(t)<0, \forall t \in[0,1]$, the previous technique can be applied whenever there is a unique positive solution of the b.v.p. (1.1). Specially, this is the case of autonomous equation because of the conservation of energy. Without going into the details let us state the theorem without the proof. 
THEOREM 8.1. Let us suppose that $\psi(t)=$ const. $<0$ and let

$$
C_{n}:=\max _{0<a_{1}<\ldots<a_{n}<1} \Phi\left(u_{a}\right) .
$$

Assume $\lambda<\lambda_{n+1}$. Then

i) $C_{n}$ is the critical value of $\left.\Phi\right|_{\Sigma}$ and $0<C_{n-1}<C_{n}$.

ii) Let $C_{n}=: \Phi\left(u_{a}\right)$. Then $u_{a}$ is a critical point of $\left.\Phi\right|_{\Sigma}$ if it changes the sign at each nodal point and is unique up to the sign.

The proof is exactly the same as the proof of Theorem 6.3. Some difficulties may arise in the construction of the path $\gamma(t)$ in the proof of Theorem 6.3 which can be avoided using the implicit function theorem.

REMARK 8.2. The Corollary (7.2) is true also if $\psi(t) \equiv-1$. In that case one should take the absolute value of $|\psi|$ instead of $\psi$ in the problems $(\mathcal{P}),\left(\mathcal{P}_{a}\right),\left(\mathcal{P}_{1-a}\right)$.

\section{ACKNOWLEDGEMENTS}

A variant of this results was a part of author's doctoral thesis. I am grateful to H. Berestycki for comments and suggestions which improved the quality of the paper. Variational approach in the Theorem 5.4 was suggested by him in a personal communications.

\section{Appendix A. Deformation Lemma}

We state the following version of Deformation Lemma. The proof can be found in Rabinowitz [14].

Deformation Lemma 1. Let $\Sigma$ be a Hilbert manifold and $\Phi \in C^{1}(\Sigma, \mathbb{R})$, satisfying (PS)-condition. If $c \in \mathbb{R}, \bar{\varepsilon}>0$, and $\mathcal{O}$ is any neighborhood of $K_{c}$, then there exists an $\varepsilon \in(0, \bar{\varepsilon})$ and $\eta \in C([0,1] \times \Sigma, \Sigma)$ such that

1. $\eta(0, u)=u$ for all $u \in \Sigma$.

2. $\eta(t, u)=u$ for all $t \in[0,1]$ if $\Phi(u) \notin[c-\bar{\varepsilon}, c+\bar{\varepsilon}]$.

3. $\eta(t, u)$ is a homeomorphism of $\Sigma$ onto $\Sigma$ for each $t \in[0,1]$.

4. dist $(\eta(t, u), u) \leq 1$ for all $t \in[0,1]$ and $u \in \Sigma$.

5. $\Phi(\eta(t, u)) \leq \Phi(u)$ for all $t \in[0,1]$ and $u \in \Sigma$.

6. $\eta\left(1, \Phi_{c+\varepsilon} \backslash \mathcal{O},\right) \subset \Phi_{c-\varepsilon}$.

7. If $K_{c}=\emptyset, \eta\left(1, \Phi_{c+\varepsilon}\right) \subset \Phi_{c-\varepsilon}$.

8. If $\Phi(u)$ is even, $\eta(1, u)$ is odd in $u$.

Here $K_{c}=\left\{u \in \Sigma, \Phi(u)=c\right.$ and $\left.\Phi^{\prime}(u)=0\right\}$ and $\Phi_{c}=\{u \in \Sigma, \Phi(u) \leq c\}$, for $c \in \mathbb{R}$.

\section{REFERENCES}

[1] H. Berestycki, Le nombre de solutions de certains problèmes semi-linéaires elliptiques, J. Funct. Anal. 40 (1980), 1-29. 
[2] C.-N. Chen, Multiple solutions for a class of nonlinear Sturm-Liouville problems on the half line, J. Differential Equations 85 (1990), 236-275.

[3] C.-N. Chen, Uniqueness and bifurcation for solutions of nonlinear Sturm-Liouville eigenvalue problems, Arch. Rational Mech. Anal. 111 (1990), 51-85.

[4] C.-N. Chen, Multiple solutions for a class of nonlinear Sturm-Liouville problems when nonlinearities are not odd, J. Differential Equations 89 (1991), 138-153.

[5] E. A. Coddington and N. Levinson, Theory of ordinal differential equations, McGrawHill, New York, 1955.

[6] C. V. Coffman, A minimum-maximum principle for a class of non-linear integral equations, J. Analyse Math. 22 (1969), 391-419.

[7] C. V. Coffman, On variational principles for sublinear boundary value problems, J. Differential Equations 17 (1975), 46-60.

[8] H. P. Heinz, Free Ljusternik-Schnirelman theory and the bifurcation diagrams of certain singular nonlinear problems, J. Differential Equations 66 (1987), 263-300.

[9] H. P. Heinz, Nodal properties and bifurcation from the essential spectrum for a class of nonlinear Sturm-Liouville problems, J. Differential Equations 64 (1986), 79-108.

[10] H. P. Heinz, Nodal properties and variational characterizations of solutions to nonlinear Sturm-Liouville problems, J. Differential Equations 62 (1986), 299-333.

[11] H. P. Heinz, Free Ljusternik-Schnirelman theory and the bifurcation diagrams of certain singular nonlinear problems, J. Differential Equations 66 (1987), 263-300.

[12] J. A. Hempel, Multiple solutions for a class of nonlinear boundary value problems, Indiana University Mathematical Journal 20 (1970/1971), 983-996.

[13] Z. Nehari, Characteristic values associated with a class of non-linear second-order differential equations, Acta. Math. 105 (1961), 141-175.

[14] P. H. Rabinowitz, Minimax methods in critical point theory with application to differential equation, CBMS Reg. Conf. Ser. in Math. 65, AMS, Providence, Rhode Island, 1986.

[15] T. Weth, Spectral and variational characterizations of solutions to semilinear eigenvalue problems, Ph.D. thesis, Johannes Gutenberg-Universität, Mainz, 2001.

\section{L. Čaklović}

Faculty of Natural Sciences

Department of Mathematics

University of Zagreb

10000 Zagreb

Croatia

E-mail: caklovic@math.hr

Received: 30.6.2009.

Revised: 11.2.2010. 